\title{
The differences of corneal astigmatism in different populations and its quantitative analysis
}

\author{
Ying Zhang ${ }^{1}$, Hongmei Chen ${ }^{2}$, Yingchun Zhang ${ }^{1}$,Yanzhen Yang $^{1}$
}

1. Departement of Ophthalmology, Tengzhou Central People's Hospital, Tengzhou, Shandong, 277500PR China. 2. Departement of Anesthesiology,TengZhou Central People's Hospital, Teng Zhou, Shandong,277500, PR China.

\begin{abstract}
Objective: To analyze the differences of corneal astigmatism in different age groups.

Methods: The clinical data of the patients from the outpatient Department of Ophthalmology of the Tengzhou Central People's Hospital from April 2014 to June 2014 was screened and statistically analyzed. The corneal curvature was measured by three different instruments: Keratometer, IOL Master and Sirius corneal topography. The changes of the corneal astigmatism in different groups were evalutaed in the study.

Results: Corneal astigmatism of different groups showed no statistical difference and the average corneal astigmatism of $70 \sim 79$ age group were higher than younger groups. As the age grew, the with-the-rule astigmatism gradually reduced from $85.8 \%$ ( A Group) to $31.0 \%$ (F Group). The against-the-rule astigmatism increased from $6.7 \%$ ( A Group ) to $45.0 \%$ (F Group).

Conclusion: The proportion of the with-the-rule astigmatism gradually reduced with the growth of age. The proportion of the against-the-rule astigmatism increased significantly. The oblique astigmatism had no obvious change before the age of 40. The proportion of oblique astigmatism increased obviously at the $40 \sim 49$ group and tended to be stable. After the age of 60 , the proportion of different types of astigmatism tended to be stable.
\end{abstract}

Keywords: with-the-rule astigmatism; against-the-rule astigmatism; oblique astigmatism.

DOI: https://doi.org/10.4314/ahs.v20i2.30

Cite as: Zhang $Y$, Chen $H$, Zhang $Y$, Yang $Y$. The differences of corneal astigmatism in different populations and its quantitative analysis. Afri Health Sci. 2020; 20(2): 775-778. https:// doi.org/ 10.4314/abs.v20i2.30

\section{Introduction}

Cornea is an important refractive stroma of human eyes, which directly affects the visual function of human eyes. Astigmatism refers to the different refractive power on the meridian, which makes the light hard to form on the retina ${ }^{1}$. The astigmatism can be divided into regular astigmatism, irregular astigmatism. Moreover, regular astigmatism can be divided into againstthe-rule astigmatism, with-the-rule astigmatism and oblique astigmatism ${ }^{2}$. According to previous studies, human astigmatisms consist of corneal astigmatism
Corresponding author:
Yanzhen Yang,
Address: No. 181 Xingtan Road,
Departement of Ophthalmology,
TengZhou Central People's Hospital,
Teng Zhou, Shandong,277500, PR China
Tel/Fax:+86-0632-5512227
Email: yang_yanzhen321@163.com

and lens astigmatism. Therefore, the cornea is an important component of human eye refractive intertexture ${ }^{3,4}$. Modern cataract surgery has been becoming into refractive surgery times, and how to reduce the astigmatism and astigmatism produced in surgery has become the focus of cataract surgery physicians ${ }^{5}$. The corneal refractive powr accounts for about $3 / 4$ of the total refractive power of the human eyes and determines the refractive state of the human eyes6. It has been found that corneal astigmatism may be related to the occurrence of myopia in human eyes 7,8 , corneal refractive surgery9 11, orthokeratology lens fitting ${ }^{12}$, IOL measurement etc13 15. Therefore, the change law of corneal astigmatism is of great guiding significance to the clinic.

\section{The research object and methods \\ The research object}

A total of 518 people, including 854 eyes data were adopted from the outpatient Department of Ophthalmology of the Tengzhou Central People's Hospital from April 2014 to June 2014. The patients with in- 
formed consent and with approval of the institutional ethics committee ranged from 20 to 80 years old were enrolled. The inclusion criteria were: the exclusion of conjunctivitis, nebula, glaucoma and other eye diseases, previous history of eye surgery, history of ocular trauma and corneal contact lens wearing (except pick a mirror for three weeks), with cooperating to complete all the ophthalmic examination.

\section{Methods of examination}

All subjects received anterior eye examination, visual acuity examination, intraocular pressure examination. The corneal curvature was measured by three different instruments: Keratometer, IOL Master and Sirius corneal topography and repeated for three times.

\section{Experimental grouping}

The subjects were assigned to six groups according to the age ; $20 \sim 29$ years old were selected as A group, 120 eyes; age $30 \sim 39$ years old were selected as B group, 142 eyes; age $40 \sim 49$ years old were selected as $C$ group, 126 eyes; age $50 \sim 59$ years old were selected as
D group, 152 eyes; age $60 \sim 69$ years old were selected as E group, 176 eyes; age $70 \sim 79$ years old were selected as F group, 138 eyes.

\section{Statistical analysis}

The results of corneal astigmatism recorded by different instruments were expressed by mean \pm standard deviation. The comparison of corneal astigmatism between each subgroups were analyzed by one-way ANOVA. The comparison of axial astigmatism ratios between each subgroups were performed by chi-square test. A p value $<0.05$ were considered statistically significant. Statistical analysis was performed using IBM SPSS Statistics Version 17 software.

\section{Results}

The five different age groups (A group, B group, C group, D group, E group) had no significant difference in corneal astigmatism ( $\mathrm{p}>0.05)$. However, the average size of corneal astigmatism in the $70 \sim 79$ years old group was higher than that of the other five groups (Table 1).

Table 1: The comparisons of corneal astigmatism between each subgroups $\left({ }^{*}: p<0.05\right)$.

\begin{tabular}{lcccccc}
\hline Group & $\mathrm{A}$ & $\mathrm{B}$ & $\mathrm{C}$ & $\mathrm{D}$ & $\mathrm{E}$ & $\mathrm{F}$ \\
\hline Number & 120 & 142 & 126 & 152 & 176 & 138 \\
& $-0.79+$ & $-0.75+$ & $-0.73+$ & $-0.75+$ & $-0.71+$ & $-0.87+$ \\
Corneal astigmatism & $0.39 \mathrm{D}$ & $0.42 \mathrm{D}$ & $0.38 \mathrm{D}$ & $0.42 \mathrm{D}$ & $0.40 \mathrm{D}$ & $0.49 \mathrm{D}^{*}$ \\
\hline
\end{tabular}

As the growth of age, the with-the-rule astigmatism gradually reduced from $85.8 \%$ (A Group) to $31.0 \%$ (F Group). The against-the-rule astigmatism increased from $6.7 \%$ (A Group) to $45.0 \%$ (F Group). The oblique astigmatism had no obvious change before the age of
40. However, the proportion of oblique astigmatism increased obvious at the $\mathrm{C}$ group, and tended to be stable after the age of 40 . After the age of 60 , the proportion of different types of astigmatism among all age groups tended to be stable(Table 2).

Table 2: The comparison of axial length of astigmatism in different age groups ${ }^{*}: \mathrm{p}<0.05$ compared to the group A).

\begin{tabular}{lcccccc}
\hline Group & $\mathrm{A}$ & $\mathrm{B}$ & $\mathrm{C}$ & $\mathrm{D}$ & $\mathrm{E}$ & $\mathrm{F}$ \\
\hline Number & 120 & 142 & 126 & 152 & 176 & 138 \\
With-the-rule astigmatism & $85.8 \%(103)$ & $76.8 \%$ & $54.8 \%$ & $44.0 \%$ & $33.0 \%$ & $31.0 \%$ \\
& & $(109)$ & $(69)^{*}$ & $(67)^{*}$ & $(58)^{*}$ & $(43)^{*}$ \\
Against-the-rule astigmatism & $6.7 \%(8)$ & $14.8 \%$ & $27.0 \%$ & $34.0 \%$ & $43.8 \%$ & $45.0 \%$ \\
& & $(21)^{*}$ & $(34)^{*}$ & $(52)^{*}$ & $(77)^{*}$ & $(62)^{*}$ \\
Oblique astigmatism & $7.5 \%(9)$ & $8.5 \%(12)$ & $18.2 \%$ & $22.0 \%$ & $23.3 \%$ & $24.0 \%$ \\
& & & $(23)^{*}$ & $(33)^{*}$ & $(41)^{*}$ & $(33)^{*}$ \\
\hline
\end{tabular}




\section{Discussion}

Corneal astigmatism is an important clinical parameter in ophthalmology and plays an important role in the diagnosis and treatment of many eye diseases. Therefore, it is meaningful to identify the changes of corneal astigmatism among the age groups..

We found no significant association between corneal astigmatism and age. However, there was a trend in growth after age of 70 (no statistical difference). The proportion of astigmatism axis changed with age. The proportion of oblique astigmatism increased significantly before age of $40 \sim 50$ and remained stable later. The proportion of against-the-rule astigmatism increasing before age of 60 , However the axial proportion of various astigmatisms tended to be stable and remained unchanged after age of 60 . In general, the human eyes gradually changed from with- the-rule astigmatism to against-the-rule astigmatism with age and this change was mainly in human corneal ${ }^{17}$. The opponents claimed that changes of the astigmatism were main in the lens ${ }^{16}$. Previous studies suggested that the reason for axial changes in corneal astigmatism might be due to eyelid laxity and orbital tissue atrophy that reduced the vertical compression of the cornea ${ }^{18}$. Moreover, the corneal astigmatism could decrease sensitivity of human eyes, resulting in human visual fatigue, and form-deprivation myopia ${ }^{7,8}$. In recent years, the super-transparent corneal tunnel incision was usually used in phacoemulsification, which would increase against-the- rule astigmatism ${ }^{19}$. Therefore, the location of the incision should be clearly defined according to the axial position of the corneal astigmatism preoperatively, which could reduce the rate of astigmatism caused by cataract surgery and improve the visual quality of patients after operation ${ }^{20}$.

With the occurrences of astigmatism corrected intraocular lenses (IOL) and TICL, more options had been offered for the correction of astigmatism. Parikakis EA's study ${ }^{21}$ found that in patients with cataract surgery, corneal astigmatism before operation accounted for about $15 \% \sim 25 \%$ of all astigmatisms . Traditional intraocular lenses did not correct preoperative corneal astigmatism, while astigmatism type intraocular lenses could correct it. In addition, the results also showed that the axial of astigmatism was associated with the age growth. Therefore, the axial deviation of IOL might produce new astigmatism. In this study, it found that the proportion of astigmatism axis was relatively stable after age of 60 . Therefore, we believed that the toric IOL implantation in cataract patients before age of 60 should be prudent and the long-term effect of TICL on myopic with astigmatism was also uncertain.

It was considered that there was no significant difference in corneal astigmatism between different age groups. However, the average value of corneal astigmatism in the age of $70 \sim 79$ group was higher. With the age increasing, the proportion of in-the -rule astigmatism decreased gradually and the proportion of against-therule astigmatism increased. The proportion of oblique astigmatism did not change significantly before the age of 40. After 40 years old, the proportion of oblique astigmatism increased significantly and tended to be stable. After 60 years old, the proportion of different types of astigmatism tended to be stable.

Our study had several limitations. The conclusion of this study was based on the changes of corneal astigmatism in different age groups, not the follow-up study of the same population. Therefore, further investigations were needed to make a more comprehensive conclusion.

\section{Acknowledgements}

None.

\section{Conflicts of interest}

None.

\section{References}

1. Naeser K, Savini G and Bregnhoj JF. Age-related changes in with-the-rule and oblique corneal astigmatism. Acta Opbthalmol. 2018; 96: 600-606 PubMed .

2. Yamamoto T, Hiraoka T and Oshika T. Apparent accommodation in pseudophakic eyes with refractive against-the-rule, with-the-rule and minimum astigmatism. Br J Ophthalmol. 2016; 100: 565-571 PubMed .

3. Li He. Comparison of corneal curvature by different methods in myopic eyes J. Rec Adv Ophthalmol. 2011,31(7):670 PubMed -672.

4. Zhang Kui, Li Zhihui, Lu Wenxiu. A research on clinical values of corneal topography and keratomrter in measuring astigmatism J. Chin J Ophthalmol. 1998,34(6):448 PubMed -450.

5. Gong Yan, Zhu Jinyu, Wu Yue, Wang Yuwen, Yuan Jianshu. Effect of cataract surgery with personalized corneal incision on corneal astigmatiasm J. Chin J Ophthalmol and Otorbinolaryngol. 2013,13(3):168-172.

6. De Bernardo M, Capasso L, Caliendo L, Paolercio F and Rosa N. IOL power calculation after corneal refractive surgery. Biomed Res Int 2014; 2014: 658350. 
7. Gwiazd aJ, GriceK, HeldR, et al. Astigmatism and the development of myopia in children. Vison Res. 2000; 40( 8) :1019 PubMed.

8. Chen F, Shi MG, Xu X. Acomparative analysis of corneal topography between emmetropia and myopia children. Chin J Optophthalmol. 2000; 2( 1) : 47-48.

9. Lan Changjun, Gan Yuye, Liao Xuan, et al. Correlation analysis between the average central corneal curvature and the corneal flap in LASIK with automated microkeratome J. Chinese Journal of Optometry. Ophthalmology. 2004,6(2):78 PubMed -80.

10. Leyland M. Validation of OrbscanII Posterior Corneal Curvature Measurment For Intraocularlens Powercaculation. J. Eye. 2004,18(4):357-360.

11. Pop M. Payette Y. Risk factors for night vision complaiment after Lasik for myopia. J. Ophthalmology. 2004.111:3-10.

12. Hu Shengfa, Zhou Xia, Tan Xingping, Xia Xiaobo. Efficacy of orthokeratology lens for treatment of juvenile myopic astigmatism. J. China Journal of Modern Medicine. 2015,25(18):98-100.

13. Lyall DA, Srinivasan S, Ng J, et al. Changes in corneal astigmatism among patients with visually significant cataract. J. Can J Ophthalmol, 2014,49(3):297-303.

14. Ale Magar JB, Cunningham F, Brian G. Comparison of automated and partial coherence keratom- etry and resulting choice of toric. IOLJ. Optom Vis Sci.2013,90(4):385-391.

15. Norrby S, Hirnschall N, Nishi Y, et al. Fluctuations in corneal curvature limit predictability of intraocular lens power calculations. J. Cataract Refract Surg. 2013,39(2):174-179.

16. Yang Suhong, Ren Huaming, Gan Xiaoling. Study on the relationship between astigmatism and progression of refractive error. J. China J Pract Ophthalmol, 2004,22(8):602-604.

17. Li Fengming.Chinese OphthalmolgyM.Beijing: People's Medical Publishing House.2004: 439.

18. Manuel AP Vilela, Victor D Castagno, Rodrigo D Meucci,et al. Asthenopia in schoolchildren. J.Clin Ophthalmol. 2015; 9: 1595-1603.

19. Qian Jin, Wang Jun, Kang Huijuan. Comparison of induced astigmatism after different corneal incision direction in phacoemulsification. J Ophthalmol CHN. 2005,14(1):31-33.

20. Rho CR,Joo CK.Effects of steep meridian incision on corneal astigmatism in phacoemulsification cataract Bur-geryJ. J Cataract Refract Surg,2012,38(4):666-671.

21. Parikakis EA, Chatziralli IP, Peponis VG, et, al. Toric intraocular lens implantation for correction of astigmatism in cataract patients with corneal ectasia. J. Case Rep Ophthalmol, 2013, 4(3):219-228. 